\title{
Devil's staircase of incompressible electron states in a nanotube
}

\author{
Dmitry S. Novikov* \\ Department of Electrical Engineering and Department of Physics, Princeton University, Princeton, NJ 08544
}

(Dated: December 16, 2004)

\begin{abstract}
It is shown that a periodic potential applied to a nanotube can lock electrons into incompressible states. Depending on whether electrons are weakly or tightly bound to the potential, excitation gaps open up either due to the Bragg diffraction enhanced by the Tomonaga - Luttinger correlations, or via pinning of the Wigner crystal. Incompressible states can be detected in a Thouless pump setup, in which a slowly moving periodic potential induces quantized current, with a possibility to pump on average a fraction of an electron per cycle as a result of interactions.
\end{abstract}

PACS numbers: 71.10.Pm, 85.35.Kt, 64.70.Rh

A carbon nanotube (NT) is a strongly interacting electron system known to be a host of many-body effects [1], including Luttinger liquid behavior [2]. At half-filling the NT is effectively dilute, and a crossover from the liquid to the 1d Wigner crystal [3] is expected [4].

Here we suggest that an external periodic potential can be a probe of both crystallization and Luttinger correlations. We show that incompressible electron states arise when the electron number density $\bar{\rho}$ (relative to halffilling) is commensurate with the potential period $\lambda_{\text {ext }}$ :

$$
\bar{\rho}=m_{\mathrm{tot}} / \lambda_{\mathrm{ext}}, \quad m_{\mathrm{tot}}=4 m .
$$

In Eq. (1), $m$ is the number of the NT electrons of each of the four polarizations [5] per period. To calculate excitation gaps we generalize the Pokrovsky-Talapov theory [6] for the case of the four coupled fermion modes.

In the absence of interactions, Bragg diffraction on the potential opens minigaps at integer density $(1),|m|=$ $1,2, \ldots[7]$. Interactions profoundly change the spectrum, yielding a devil's staircase of incompressible states at $r a$ tional $m=p / q$. In such a state, the NT electron system is locked by the potential into a $q \lambda_{\text {ext }}$-periodic structure. If detected, e.g. in a Thouless pump setup [7], corresponding minigaps would provide a direct probe of interactions, with a possibility to map the devil's staircase by pumping at fractions of the base frequency.

One-dimensional interacting electrons are conventionally described by the Tomonaga - Luttinger liquid [2]. This hydrodynamic approach is valid in a small momentum shell near the Fermi points, with excitations extended over the whole system. Adequate description of crystallization and commensurability requires including the curvature of the electronic dispersion that becomes important at low density. The curvature can yield crystallization or commensuration by coupling charge and spin modes and by introducing a length scale into an otherwise scale-invariant Gaussian theory. In this work we treat both electron interactions and the curvature of the dispersion non-perturbatively by making use of the relativistic Dirac spectrum of a half-filled nanotube. Curvature is controlled by the Dirac gap and is bosonized exactly by virtue of the massive Thirring - sine-Gordon duality. [8, 9] This enables us to study incompressible states both in the limit of a narrow-gap Luttinger liquid and in that of the locked Wigner crystal.

Our course of action is to introduce the bosonized description for the NT electrons, develop the phase soliton method and find excitation gaps from the renormalized sine-Gordon action, draw the phase diagram in the semiclassical limit, and comment on the experimental means to detect incompressible states.

The model. - Nanotube electrons in the forward scattering approximation [10] are described by the four flavors of Dirac fermions $\psi_{\alpha}=\left(\psi_{\alpha}^{R} \psi_{\alpha}^{L}\right)^{T}, \alpha=1-4$, whose interaction is written in terms of the smooth envelope $\rho(x)=\sum_{\alpha=1}^{4} \psi_{\alpha}^{+}(x) \psi_{\alpha}(x)$ of the total charge density. The second-quantized Hamiltonian $\mathcal{H}=\mathcal{H}_{0}+\mathcal{H}_{\mathrm{bs}}+\mathcal{H}_{\text {ext }}$, where $\mathcal{H}_{0}$ is the massless Dirac Hamiltonian

$$
\mathcal{H}_{0}=-i \hbar v \int \sum_{\alpha=1}^{4} \psi_{\alpha}^{+} \sigma_{3} \partial_{x} \psi_{\alpha} d x+\frac{1}{2} \sum_{k} \rho_{-k} V(k) \rho_{k}
$$

with the Coulomb interaction $V(k)=\frac{2 e^{2}}{\varepsilon+1} \ln \left[1+(k a)^{-2}\right]$ for a tube of radius $a$ placed on a substrate with the dielectric constant $\varepsilon$. The curvature of the electron dispersion controlled by the gap $2 \Delta_{0}$ at half-filling introduces backscattering at each NT Dirac point:

$$
\mathcal{H}_{\mathrm{bs}}=\Delta_{0} \int \sum_{\alpha=1}^{4} \psi_{\alpha}^{+} \sigma_{1} \psi_{\alpha} d x
$$

We emphasize that the backscattering $\Delta_{0}$ is not the usual interaction-induced $V\left(2 k_{F}\right)$ term (which is undetectably small in metallic NTs), but rather is present at the singleparticle level $[5,11]$ : Depending on the tube chirality, the bare gap $\Delta_{0}$ can be in the range $\Delta_{0} \lesssim 10 \mathrm{meV}$ to $\sim 0.5 \mathrm{eV}$; it can also be controlled by the parallel magnetic field [12]. Adding the periodic potential $U(x)$ and the chemical potential $\mu[\mu=0$ at half-filling $]$ results in

$$
\begin{aligned}
& \mathcal{H}_{\mathrm{ext}}=\int d x \rho(x)\{U(x)-\mu\}, \\
& U(x)=A \cos k_{\mathrm{ext}} x, \quad k_{\mathrm{ext}}=2 \pi / \lambda_{\mathrm{ext}} .
\end{aligned}
$$

Qualitatively, our findings will be valid for any realistic potential which justifies the simplest choice (5); typically, 
$\lambda_{\text {ext }} \sim 0.1-1 \mu \mathrm{m}$. The Hamiltonian $\mathcal{H}$ is $\mathrm{U}(4)$ invariant with respect to rotations in the fermion flavor space.

Bosonization of the nanotube electrons $\psi_{\alpha}=$ $\frac{1}{\sqrt{2 \pi a}} e^{i \Theta_{\alpha}}$ is exact even in the presence of (3). It maps the problem of the four interacting Dirac fermion modes onto the sine-Gordon model of the four coupled bose fields $\Theta_{\alpha}$. We rotate $[4,7]$ to the basis of the charge mode $\theta^{0}$ and three neutral modes $\theta^{a}$, in which case the charge density $\rho(x)=\frac{2}{\pi} \partial_{x} \theta^{0}$, and the Gaussian action (bosonized $\mathcal{H}_{0}$ ) is diagonal $[\hbar=v=1]$ :

$$
\mathcal{L}_{0}=\frac{1}{2 \pi} \int d x\left(\left(\partial_{t} \theta^{0}\right)^{2}-K\left(\partial_{x} \theta^{0}\right)^{2}+\sum_{a=1}^{3}\left(\partial_{\mu} \theta^{a}\right)^{2}\right) .
$$

The slow momentum dependence of the charge stiffness $K_{k}=1+4 \nu V(k), \nu^{-1}=\pi \hbar v$, is irrelevant, and we take it as constant $K \equiv K_{k \sim 1 / l_{\mathrm{ch}}}, l_{\mathrm{ch}}$ being the size of the charged-mode soliton (described below). Assuming $l_{\mathrm{ch}} \sim l_{s}$, with the screening length $l_{s} \sim 1 \mu \mathrm{m}$, and using $e^{2} / \hbar v \simeq 2.7$, one estimates $K \simeq 40$ for for the standalone tube; $K \simeq 10$ if the tube is placed on a substrate with dielectric constant $\varepsilon \simeq 10$. The logarithmic behavior of $V(k)$ underlies the fact that the Coulomb interaction $V(x) \propto 1 /|x|$ is essentially local in one dimension, $\frac{1}{2} \int d x d x^{\prime} \rho(x) V\left(x-x^{\prime}\right) \rho\left(x^{\prime}\right) \simeq \frac{\hbar v}{2 \pi} \int d x K\left(\partial_{x} \theta^{0}\right)^{2}$.

The nonlinear part of the sine-Gordon Lagrangian [coming from $\left.\mathcal{H}_{\mathrm{bs}}\right]$ reads $[4,7,13]$

$$
\begin{aligned}
& \mathcal{L}_{\mathrm{bs}}=-\int d x g_{0} \mathcal{F}\left(\theta^{0}+2 \tilde{\mu} k_{\mathrm{ext}} x-2 \tilde{A} \sin k_{\mathrm{ext}} x, \theta^{a}\right), \\
& \mathcal{F}\left(\theta^{0}, \theta^{a}\right)=\cos \theta^{0} \cdot \prod_{a=1}^{3} \cos \theta^{a}+\sin \theta^{0} \cdot \prod_{a=1}^{3} \sin \theta^{a},
\end{aligned}
$$

where $g_{0}=4 \Delta_{0} / \pi D a^{2}$, and $D \simeq \hbar v / a$ is the $1 \mathrm{~d}$ bandwidth. In Eq. (7) we included the coupling (4) to external fields by shifting the charge mode $\theta^{0} \rightarrow \theta^{0}-$ $\frac{2}{\hbar v} \int^{x} K^{-1}(U-\mu) d x^{\prime}$, with $\tilde{\mu}=\mu /\left(K \epsilon_{0}\right), \tilde{A}=A /\left(K \epsilon_{0}\right)$, $\epsilon_{0}=\hbar k_{\text {ext }} v$.

In what follows, it is useful to first describe elementary excitations of the stand-alone tube, $U \equiv 0$. As shown by Levitov and Tsvelik [4], in the bosonized language adding one electron corresponds to a composite soliton of both the charge and the flavor modes. In such a composite object, the charge mode changes by $\pi / 2$ (adding unit charge) over the length $l_{\mathrm{ch}}$, whereas the neutral sector adds a particular SU(4) flavor to the electron by means of the solitons of $\theta^{a}$ "switching" by $\pm \pi / 2$ on a shorter scale $l_{\mathrm{fl}} \sim K^{-1 / 2} l_{\mathrm{ch}}$, right in the middle of the charge soliton. The composite soliton is a unit charge configuration of the minimal energy, obtained by optimizing the action $\mathcal{L}_{0}+\mathcal{L}_{\text {bs }}$ in the limit of large Coulomb repulsion $K \gg 1$. Technically, such an optimization results in the soft neutral modes $\theta^{a}$ adjusting to create the effective potential $\overline{\mathcal{F}}\left(\theta^{0}\right)=\min _{\left\{\theta^{a}\right\}} \mathcal{F}\left(\theta^{a}, \theta^{0}\right) \sim \cos 4 \theta^{0}$ for the stiff charge mode $\theta^{0}$.

In the absence of external potential, $U \equiv 0$, the system $\mathcal{L}_{0}+\mathcal{L}_{\text {bs }}$ describes the Wigner crystal - Luttinger liquid crossover. In particular, raising the chemical potential from half-filling [band insulator, or the "Dirac vacuum"] to just above the charge gap produces the train of weakly overlapping composite solitons described above $\left[\bar{\rho} l_{\mathrm{fl}} \ll 1\right.$, "Wigner crystal"], in which overlapping charge solitons maintain a quasi-long-range order. Further increase of $\mu$ leads to strongly overlapping solitons rendering the nonlinear term (7) irrelevant $\left[\bar{\rho} l_{\mathrm{fl}} \gg 1\right.$, Luttinger liquid]. We emphasize that the crystal - liquid crossover occurs due to the finite soliton size $l_{\mathrm{fl}} \propto \Delta_{0}^{-\zeta}$ that scales inversely with the curvature of the dispersion. Curvature is also responsible for binding flavor to charge through the term (7). The $U \equiv 0$ system is compressible [4].

Periodic potential locks electrons into incompressible states. Technically, the term (7) becomes relevant whenever the density $2 \tilde{\mu}=m$ [Eq. (1)] is integer [7]. When $m$ is a simple fraction, $2 \tilde{\mu}=p / q$, to identify incompressible states one utilizes the phase soliton method [6]. We generalize it for the case of the four nanotube modes by expanding in powers of the coupling $g_{0}$ as $\theta^{j}=\bar{\theta}^{j}+g_{0} \theta^{j(1)}+\ldots+g_{0}^{n} \theta^{j(n)}+\ldots, j=0, a$, and finding the effective Lagrangian $\mathcal{L}_{m}\left[\bar{\theta}^{0}, \bar{\theta}^{a}\right]$ for the phase modes $\bar{\theta}^{0}$ and $\bar{\theta}^{a}, a=1-3$, to the lowest order in $g_{0}$. The phase modes $\bar{\theta}^{0}, \bar{\theta}^{a}$ are constant in the commensurate phase, whereas an excitation is a composite phase soliton, in which the phase fields $\bar{\theta}^{0}(x), \bar{\theta}^{a}(x)$ describe a slow deformation of the regular commensurate configuration. Excitation gap is given by the energy of the composite soliton, renormalized by quantum fluctuations.

We note that it is the curvature $\propto \Delta_{0}$ that yields incompressible states. Same is true for the non-interacting electrons: When $\Delta_{0}=0$, the external potential is gauged away from the Hamiltonian $-i \hbar v \sigma_{3} \partial_{x}+U(x)$.

The composite phase soliton is a result of optimization of the corresponding effective action $\mathcal{L}_{m}\left[\bar{\theta}^{0}, \bar{\theta}^{a}\right]$ (examples of which are given below). This problem will be similar to the $U \equiv 0$ case described above: When interaction is strong, $K \gg 1$, the neutral phase modes $\bar{\theta}^{a}$ adjust [on the scale $\overline{l_{\mathrm{f}}}$ ] to create an effective potential for the charged phase mode $\bar{\theta}^{0}$. The crucial difference from the former case is that this optimization will qualitatively depend on whether the system is in the regime of the Luttinger liquid or in that of the Wigner crystal. In this sense, periodic potential naturally distinguishes between the opposite sides of the crystal - liquid crossover, by bringing about the additional length scale, its period $\lambda_{\text {ext }}$. Technically, the saddle point of $\mathcal{L}_{m}$ will depend on whether the neutral modes are adiabatic or fast on the length scale on which the effective potential (7) changes appreciably. Below we consider both cases separately.

Bragg diffraction in a four-flavor Luttinger liquid.In the adiabatic limit $\overline{l_{\mathrm{f}}} \gg \lambda_{\text {ext }}$ of extended flavor excitations, exchange is important: The system (correlated over many $\lambda_{\text {ext }}$ ) "knows" that it is comprised of particles of the four different flavors. This limit is connected to the non-interacting case, where particles repel only due to the Pauli principle. For integer density $m$, averag- 
ing (7) over the period $\lambda_{\text {ext }}$ reduces the problem to the $U \equiv 0$ case with $\mathcal{L}_{m}=\mathcal{L}_{0}+\mathcal{L}_{\text {bs }}, g_{0} \rightarrow g_{0} J_{m}(2 \tilde{A})$, yielding the $K \gg 1$ minigaps $\Delta_{m} \simeq K^{1 / 2} D^{1 / 5}\left|\Delta_{m}^{(0)}\right|^{4 / 5}$ that are enhanced by the quantum fluctuations compared to the noninteracting values $\Delta_{m}^{(0)}=2 \Delta_{0} J_{m}(2 \tilde{A})$ [7].

The fractional $m=\frac{1}{2}$ case is considered in detail in Ref. 13. A somewhat lengthy calculation yields the effective action of the form $\mathcal{L}_{1 / 2}=\mathcal{L}_{0}+$ $v(\tilde{A}) \sum_{a} \cos 2 \bar{\theta}^{a} \cos 2 \bar{\theta}^{0}-u(\tilde{A}) \sum_{a>b} \cos 2 \bar{\theta}^{a} \cos 2 \bar{\theta}^{b}$. We find the charge excitation gap $\Delta_{1 / 2} \propto \frac{K-1}{\sqrt{K}} D\left(\frac{\Delta_{0}}{\epsilon_{0}}\right)^{2}$ (that vanishes in the noninteracting limit $K \rightarrow 1$ in accord with the Bloch theory), whereas flavor excitations are governed by the $\mathrm{SU}(4) \simeq \mathrm{O}(6)$ Gross - Neveu Lagrangian [14] that can be written in terms of six Majorana fermions $\chi \sim e^{i \theta}, \mathcal{L}_{\mathrm{GN}}=i \bar{\chi}_{j} \gamma_{\mu} \partial_{\mu} \chi_{j}-$ $u(\tilde{A})\left(\bar{\chi}_{j} \chi_{j}\right)\left(\bar{\chi}_{j^{\prime}} \chi_{j^{\prime}}\right)$. Although the charge gap $\Delta_{1 / 2}$ vanishes for certain values of the potential amplitude (zeroes of $v(\tilde{A})$ given in Ref. 13), the excitation gap never closes due to flavor, since the Gross - Neveu coupling $u(\tilde{A}) \neq 0$.

Locked Wigner crystal. - Below we focus on the opposite limit $\bar{l}_{\mathrm{fl}} \ll \lambda_{\text {ext }}$, in which the charge mode dominates, whereas exchange (overlap of the flavor solitons) is relatively unimportant. We will show that the effective description in this regime is that of a single mode of fermions with the density (1), locked by the external potential. Physically this happens since when the electron wavefunctions (represented by solitons) are localized on the scale $\lambda_{\text {ext }}$, Coulomb repulsion wins over exchange. Naturally, in this case the appropriate saddle point of $\mathcal{L}_{0}+\mathcal{L}_{\text {bs }}$ is equivalent to the semi-classical description, with the WKB condition $\overline{l_{\mathrm{fl}}} \ll \lambda_{\text {ext }}$. Technically, the neutral mode "switching" produces the effective charge mode potential with a quarter-period, similarly to the $U \equiv 0$ case above: $g_{0} \mathcal{F}\left(\theta^{0}+m k_{\text {ext }} x-2 \tilde{A} \sin k_{\text {ext }} x, \theta^{a}\right) \sim$ $g \cos \left(4 \theta^{0}+m_{\text {tot }} k_{\text {ext }} x-8 \tilde{A} \sin k_{\text {ext }} x\right)$. This potential now depends on the total density $m_{\text {tot }}$, Eq. (1).

To obtain the renormalized coupling $g$ we utilize adiabaticity of $U(x), \overline{l_{\mathrm{fl}}} \ll \lambda_{\text {ext }}$. The renormalization group produces the effective coupling $g$ on the scales $a<l<l_{\mathrm{fl}}$ that appear "microscopic" for the external potential. Thus the neutral modes can be integrated out, yielding both $l_{\mathrm{fl}}$ and $g$ independent of the shape of the potential $U(x)$. The problem becomes similar to the $U \equiv 0$ case. The coupling $g$ follows from the scaling $g \simeq g_{0}\left(l_{\mathrm{fl}} / a\right)^{-3 / 4}$ and self-consistency $g\left(l_{\mathrm{fl}}\right) \sim 1 / l_{\mathrm{fl}}{ }^{2}$. $[4,7,13]$

Re-fermionization. - Let us now complete our singlemode description by mapping the system in the limit $\overline{l_{\mathrm{fl}}} \ll \lambda_{\text {ext }}$ onto the problem of spinless Dirac fermions in the external potential (5). We introduce the displacement field $\Theta=2 \theta^{0}$ for the total density $\rho \equiv \frac{1}{\pi} \partial_{x} \Theta$. To preserve commutation relations, we rescale the canonical momentum $\Pi_{\Theta}=\frac{1}{2} \Pi_{\theta^{0}}$. Changing variables in the Lagrangian $\frac{1}{2 \pi}\left\{K\left(\partial_{x} \theta^{0}\right)^{2}+g \cos \left(4 \theta^{0}+\ldots\right)\right\}$, we obtain the effective Lagrangian for the charge mode $\Theta$,

$$
\begin{gathered}
\mathcal{L}_{\text {eff }}[\Theta]=\frac{\hbar v^{\prime}}{\pi} \int d x\left\{\frac{1}{2 v^{\prime 2}}\left(\partial_{t} \Theta\right)^{2}-\frac{K^{\prime}}{2}\left(\partial_{x} \Theta\right)^{2}\right. \\
\left.-g \cos \left(2 \Theta+m_{\text {tot }} k_{\text {ext }} x-2 \tilde{A}^{\prime} \sin k_{\text {ext }} x\right)\right\}
\end{gathered}
$$

with the rescaled parameters $v^{\prime} \equiv 4 v, K^{\prime} \equiv K / 16$, and $\tilde{A}^{\prime} \equiv A / K^{\prime} \hbar k_{\text {ext }} v^{\prime}=4 \tilde{A}$. The velocity quadrupling corresponds to counting incoming fermions regardless of their (four) flavors. With the same accuracy that allowed us to neglect the flavor sector, the rescaled value $K^{\prime} \approx 1+(K-1) / 16 \equiv 1+\nu^{\prime} V(q)$ is by definition the charge stiffness for the spinless Dirac fermions of velocity $v^{\prime}$, with the density of states $\nu^{\prime}=1 / \pi \hbar v^{\prime}$. The external fields $U$ and $\mu$ are not rescaled. Introducing the Dirac spinors $\Psi=\frac{1}{\sqrt{2 \pi a^{\prime}}} e^{i \Theta}$ relative to the new cutoff $a^{\prime} \sim l_{\mathrm{f}}$, we obtain the effective Hamiltonian

$$
\begin{aligned}
\mathcal{H}_{\mathrm{eff}}[\Psi]= & \int d x \Psi^{+}\left\{-i \hbar v^{\prime} \sigma_{3} \partial_{x}+\Delta^{\prime} \sigma_{1}+U(x)\right. \\
& -\mu\} \Psi+\frac{1}{2} \sum_{k} \rho_{-k} V(k) \rho_{k}
\end{aligned}
$$

for the fictitious spinless Dirac fermions of unit charge, with the number density $\rho(x)=\Psi^{+} \Psi$, coupled to the external fields in the natural way, Eq. (4). The fermions $\Psi$ represent the original NT electrons traced over the flavor states. The effective gap $\Delta^{\prime}$ in Eq. (10) corresponds to the renormalized coupling $g$ entering the Lagrangian (9) by $g \simeq \frac{\Delta^{\prime}}{\hbar v^{\prime} a^{\prime}}$, yielding $\Delta^{\prime} \simeq \frac{\hbar v^{\prime}}{l_{\mathrm{fl}}} \simeq D^{1 / 5} \Delta_{0}^{4 / 5}$.

The (charge) excitation gaps are now estimated via the phase soliton approach [6] for the single-mode system (9) and (10), yielding the devil's staircase. For integer $m_{\text {tot }}= \pm 1, \pm 2, \ldots$, in the classical limit $K \rightarrow \infty$, we find

$$
\Delta_{m} \simeq K^{1 / 2}\left|J_{m_{\text {tot }}}\left(2 \tilde{A}^{\prime}\right)\right|^{1 / 2} D^{1 / 5} \Delta_{0}^{4 / 5} .
$$

The effective density quadrupling $m \rightarrow m_{\text {tot }}$ in (11) is due to strong interactions: Electrons of all flavors avoid each other, their ground state being a Slater determinant of the quadrupled size.

Phase diagram. - The Hamiltonian (10) in the limit $K \gg 1$, coarse-grained beyond $l_{\mathrm{f}}$, describes the classical chain of $n_{e}$ electrons and $n_{h}$ holes per period, $m_{\mathrm{tot}}=n_{e}-n_{h}$. Their positions $x_{i}$ in the minima and $y_{j}$ in the maxima of $U(x)$, as well as the characteristic Coulomb blockade hexagons labeled by $\left(n_{e}, n_{h}\right)$ in Fig. 1, are obtained by minimizing $E_{\mathrm{cl}}(A) / N-\mu\left(n_{e}-n_{h}\right)$, where

$$
\begin{gathered}
E_{\mathrm{cl}}=\sum\left[\Delta^{\prime}+U\left(x_{i}\right)\right]+\sum\left[\Delta^{\prime}-U\left(y_{j}\right)\right] \\
+\sum_{i>i^{\prime}} V\left(x_{i}-x_{i^{\prime}}\right)+\sum_{j>j^{\prime}} V\left(y_{j}-y_{j^{\prime}}\right)-\sum_{i, j} V\left(x_{i}-y_{j}\right)
\end{gathered}
$$

for a finite tube of the length $L=N \lambda_{\text {ext }}$. In Fig. 1, the hexagon size is controlled by the NT capacitance per period, $C_{0} \simeq \frac{\lambda_{\text {ext }}}{2 \ln \left(l_{s} / \lambda_{\text {ext }}\right)}$. For $n_{e}, n_{h} \gg 1$, the Thomas - 


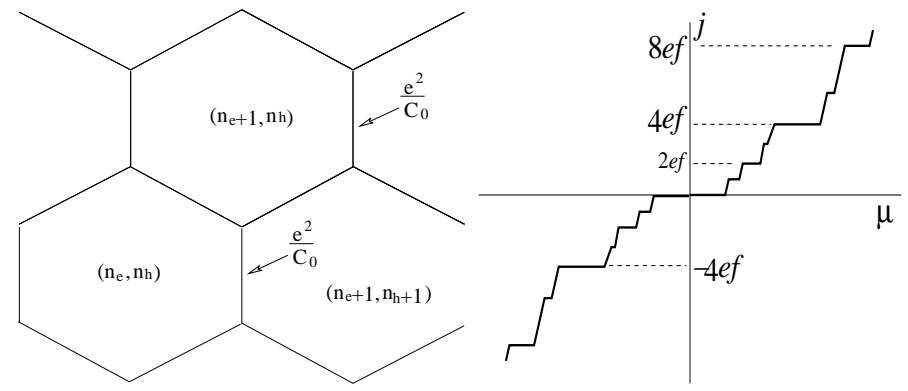

FIG. 1: Left: Phase diagram in the $(A, \mu)$ plane according to the model (12). In the limit $n_{e}, n_{h} \gg 1$ the period in $A$ becomes $e^{2} / C_{1}$. Right: Mapping the devil's staircase in the Thouless pump setup. In addition to the integer- $m$ plateaus, the ones with fractional $m$ appear due to electron interactions.

Fermi approximation $\rho(x) \approx \rho_{\mathrm{TF}}(x) \propto U(x)$ estimates the interaction energy inside each half-period ("quantum dot") as $\frac{e^{2}}{2} \int d x d x^{\prime} \frac{\rho_{\mathrm{TF}}(x) \rho_{\mathrm{TF}}\left(x^{\prime}\right)}{\left|x-x^{\prime}\right|} \equiv \frac{n_{e}^{2} e^{2}}{2 C_{1}}$, where the "dot capacitance" $C_{1} \simeq \frac{\lambda_{\text {ext }}}{2 \pi \ln \left(\lambda_{\text {ext }} / a^{\prime}\right)}$. In this limit, all the hexagon domains of the phase diagram become identical with the width $e^{2} / C_{1}$. Remarkably, this period asymptotically follows from Eq. (11) with $\tilde{A}^{\prime} \approx \frac{\pi}{2} \cdot \frac{A}{e^{2} / C_{1}}$ $\left[K \equiv K_{1 / \lambda_{\text {ext }}} \approx K-1\right]$, using the Bessel function zeroes $2 \tilde{A}_{m_{\text {tot }}}^{\prime(n)} \approx \frac{3 \pi}{4}+\frac{\pi m_{\text {tot }}}{2}+\pi n, n=\min \left\{n_{e}, n_{h}\right\}$. The borders between the domains correspond to the incompressible states with fractional $n_{e}$ and $n_{h}$ (not shown).

Quantized current. - The devil's staircase can be mapped in the Thouless pump setup [7]. With the Fermi level in the gap, a slowly moving wave $U(x-s t)$ with the frequency $f=s / \lambda_{\text {ext }}$ will generate the quantized current $j=m_{\text {tot }} e f$. The moving potential can be created by gating, optical methods, or acoustic field. Estimated gap values $\Delta_{m}$ in the meV range ensure adiabaticity leading to current quantization and possible metrological applications. Novel incompressible states with fractional $m$ would correspond to the current quantized in the fractions of $4 e f$, as illustrated in Fig. 1. Current changes sign at half-filling due to the Dirac symmetry.

Conclusions. - We demonstrated that coupling to a periodic potential results in the devil's staircase of incompressible electron states. Excitation gaps are found in the limit of the narrow-gap Luttinger liquid and in that of the Wigner crystal, by selecting the saddle point of the nonlinear action of the four bosonic modes. When the Coulomb interaction dominates, the system behaves as a single fermion mode with renormalized mass and velocity. Control over the NT gap, the screening length, and the parameters of the potential makes this setup a unique probe of the Luttinger liquid - Wigner crystal crossover and of commensuration effects in 1d. Novel effect of adiabatic pumping at fractions of base frequency is linked to the interaction-induced incompressible states. The effective single-mode description could also rationalize recent manifestations of Wigner crystallization in transport, such as the $e^{2} / h$ steps in conductance [15].

This work was supported by NSF MRSEC grant DMR 02-13706.

* Electronic address: dima@alum.mit.edu

[1] J. Nygard, D.H. Cobden, M. Bockrath, P.L. McEuen, P.E. Lindelof, Appl. Phys. A 69, 297 (1999)

[2] M. Stone (Ed.), Bosonization, World Scientific, Singapore (1994)

[3] L.I. Glazman, I.M. Ruzin, and B.I. Shklovskii, Phys. Rev. B 45, 8454 (1992); H.J. Schulz, Phys. Rev. Lett. 71, 1864 (1993)

[4] L. S. Levitov, A. M. Tsvelik, Phys. Rev. Lett. 90, 016401 (2003)

[5] R. Saito, G. Dresselhaus and M. S. Dresselhaus, Physical Properties of Carbon Nanotubes, Imperial College Press, London, 1998.

[6] V.L. Pokrovsky, A.L. Talapov, Sov. Phys. JETP 75, 1151 (1978); P. Bak, Rep. Prog. Phys. 45, 587 (1982); J. Frenkel and T.A. Kontorova, Zh. Eksp. Teor. Fiz. 8, 1340 (1938)

[7] V.I. Talyanskii, D.S. Novikov, B.D. Simons, L.S. Levitov, Phys. Rev. Lett. 87, 276802 (2001)

[8] S. Coleman, Phys. Rev. D 11, 2088 (1975)

[9] F.D.M. Haldane, J. Phys. A 15, 507 (1982)

[10] Y.A. Krotov, D.-H. Lee, S.G. Louie, Phys. Rev. Lett. 78, 4245 (1997); R. Egger, A. O. Gogolin, Phys. Rev. Lett. 79, 5082 (1997); C. Kane, L. Balents, M. P. A. Fisher, Phys. Rev. Lett. 79, 5086 (1997); A. Odintsov and H. Yoshioka, Phys. Rev. Lett. 82, 374 (1999)

[11] C. L. Kane and E. J. Mele, Phys. Rev. Lett. 78, 1932 (1997); C. Zhou, J. Kong, and H. Dai, Phys. Rev. Lett. 84, 5604 (2000); M. Ouyang, J.L. Huang, C.L. Cheung, C.M. Lieber, Science 292, 5517 (2001)

[12] H. Ajiki and T. Ando, J. Phys. Soc. Jpn. 65, 505 (1996); J. -O. Lee, J. R. Kim, J. J. Kim, J. Kim, N. Kim, J. W. Park, and K. H. Yoo, Sol. Stat. Comm. 115, 467 (2000); U.C. Coskun, T.-C. Wei, S. Vishveshwara, P.M. Goldbart, A. Bezryadin, Science 304, 1132 (2004)

[13] D.S. Novikov, preprint cond-mat/0407498

[14] D.J. Gross and A. Neveu, Phys. Rev. D 10, 3235 (1974)

[15] S. Frank, P. Poncharal, Z.L. Wang, and W.A. de Heer, Science 280, 1744 (1998); M.J. Biercuk, N. Mason, J. Martin, A. Yacoby, C.M. Marcus, Phys. Rev. Lett. 94, 026801 (2005); K.A. Matveev, Phys. Rev. Lett. 92, 106801 (2004) 